\title{
Preliminary Framework for Studying Self-reported Data in Electronic Medical Records within a Continuing Care Retirement Community
}

\author{
Kelley Gurley and Anthony F. Norcio \\ Department of Information Systems \\ University of Maryland Baltimore County (UMBC) \\ Baltimore, MD 21250 USA \\ $\{$ kgurley1, norcio\} @umbc. edu
}

\begin{abstract}
This paper serves as a preliminary framework design for studying data that is reported by patient's living within a retirement community and how this impacts the quality of care that is received when using a telemonitoring device. It reviews concepts that are involved in usage of this type of technology and presents some initial research questions in studying this population.
\end{abstract}

Keywords: Telemonitoring, Telehealth, Aging Population, Electronic Medical Record, Home Care, Continuing Care Retirement Community.

\section{Introduction}

Due to the new government incentives to increase the use of electronic medical records and devices that support them, many people already have access to devices that will update their health records remotely. An electronic medical record (EMR) is an electronic version of a person's medical health record. Devices that support the ability of a patient to upload information directly to that medical record are usually referred to as Telehealth or Telemonitoring devices. According to the Centers for Medicare and Medicaid Services (CMS), Telehealth (or Telemonitoring) is the use of telecommunications and information technology to provide access to health assessment, diagnosis, intervention, consultation, supervision and information across distances [1]. The value of such devices is to support longer independent living for ageing adults [2].

The use of electronic medical records in this study will be focused within the confines of a continuing care retirement facility. The patients within these communities are referred to as residents, as they reside at the specific community. These medical records are usually updated by the care team for that resident. The care team consists of all persons that assess, treat, diagnose or impact the resident's health care. These include, but are not limited to their physicians, nurses, care coordinators, rehabilitation team, etc.

With constant changes to medication, many residents are requested to keep track of certain vitals in order to assist the care team in ensuring that the medicine which has been prescribed is working as intended. An example that is common in the medical community occurs with changes to high blood pressure medications and the request of 
the resident to keep track of their blood pressures for a given time, sometimes at specific times during the day. This information assists the physicians in determining changes that may be needed to improve the resident's health. It is at this time that medical record gets updated with information that is obtained from the resident. Another example would be a diabetic resident, tracking their blood sugars levels, similar to the blood pressure check, it would occur at specific intervals as determined by the resident's health care providers.

As with any information that is being maually documented, there is always a risk of data entry error. A suitable more efficient way would be for information to be automatically updated when taken by the resident. Using remote health monitoring in situations like these can be ideal for both the resident and the care team. This information, as mentioned earlier is used to assist with treating the resident, therefore, it is critical that it is accurate.

\section{Background}

In order for telehealth to be useful and successful within a retirement community setting, it must be perceived as useful by the organization, health care team and the residents. Usefulness from an organization perspective will be associated with the reduction of cost and improvement of efficiencies and effectiveness [3]. Usefulness for the health care team would provide assistance with the resident's compliance to health care protocols and a reduction of hospitalizations and readmissions. Usefulness defined by the resident would assist with increased ownership of their personal health care.

In order for an organization to invest in telehealth technologies, some costeffectiveness will be considered. One review of 50 studies which include different telemedicine applications within a variety of departments found results that ranged from a $400 \%$ reduction in referrals for urgent assessment to no major differences in clinical outcomes [4]. This information demonstrates the inconsistency in data collected in this field due to the technology being new and not having a huge amount of data to assess its true cost effectiveness that could compare currently used protocols against the telehealth alternative.

Another key barrier for adoption and development of telehealth applications are the licensure requirements. This is governed independently by each state and has been difficult to get "buy in" from all states to allow a physician the ability to practice telemedicine [5]. The other common barriers are malpractice and the reimbursement of those providers that provide telemedicine. The high cost of telecommunications has also affected its growth. Once considerations have been made to accommodate some of these issues there will be a greater interest in performing more cost-effective reviews of this type of technology.

To support the use of telehealth within an organization for the health care team, this technology should be able to demonstrate improvement of care for the resident [6]. A couple of examples can be provided by research studies which demonstrate the improvement of patient adherence to medication of $50 \%$ when the use of an automated telephone patient monitoring application was used [7]. Another would be a $0.3 \%$ lower HbA1c level that demonstrates better glycemic control by patients who 
used an automated telephone disease management application [8]. To support the adoption of telehealth by the residents it should be easy to use [9].

\section{Technology}

Understanding how technology is viewed and used by different groups may assist with determining how it may be adopted. Some factors that will be reviewed that may play a role with groups are: gender, age, and experience with technology as these may determine the accuracy of data that is entered, depending on the user's ability to understand and use technology [10]. People's attitudes towards technology are generally an effect of their experiences with technology in general. A person that has worked on a computer for the last 30 years of their work life prior to retiring will probably have a different perspective than someone who has retired and never used a computer $[11,12]$.

\subsection{Gender}

Some research has demonstrated that genders have different perspectives when it comes to technology $[13,14,15]$. Men need to understand how it is going to be useful whereas women need to know that it will be easy to use, along with peer approvals and support. Once these differences are understood, then it is easier to determine how to educate the users of the new application.

\subsection{Age}

Age plays a major factor in user acceptance and usage of technology [16]. There are several psychological factors that affect learning and retention. These will need to be considered when evaluating teaching, training and accurate use of a telemonitoring system for people 65 years and older.

\subsection{Experience}

Additional measures of past exposure and use of technology will need to be considered as this may determine the speed of grasping new technologies. It may also be used to explain negative or positive feelings [17] towards the telemonitoring device.

\subsection{Device Components}

The system being studied is divided into 2 different components. The first component is a portable device that is placed in the resident's home. Connections are available for specific brands/types of blood pressure devices, pulse oximeter and blood glucose devices. There is also availability for wirelessly connected, blue tooth enabled weight scales. The device allows for manual entry through a touch screen input. The second component is the back end database which the device transmits information to and is managed by the care team. The care team can determine intervals for when the resident should participate in an activity. These range from watching videos, answering 
questions regarding well being and medication adherence along with taking a reading from the specific devices.

All decisions are made by the care team with respect to what information is needed, intervals etc. The device also allows the care team to contact the resident via video conferencing. This requires high speed internet connectivity in order to be maintain a stable video session. All information that is entered by the resident gets uploaded after each session into the database which is viewed by the care team. This part of the telehealth system allows for tracking and collecting information. It allows the health care team to generate reports of the resident data over time periods and displays the information in text form and through the use of graphs.

\section{Study Purpose}

A telehealth product must be deemed reliable and accurate before the health care team would consider it for use [18]. If information is not accurate it will negatively impact the decisions that are made by the health care team and the care of the resident. The telehealth device being studied, allows manual entry of several health measures including blood pressure and blood glucose levels. These measures, could also be uploaded directly from specified blood pressure monitor and blood glucose devices. It also transmits all entries automatically at the end of every session and stores all data being captured. The device also allows the healthcare team to initiate video conferencing to the resident.

The providers can also establish threshold violations for each resident specific to their needs. There is also a feature to immediately contact the health care team if the data that is captured is above or below a specific threshold value. The device also provided the resident with videos on chronic disease management that is related to their medical need. These devices can also remind residents' to participate in a specific activity i.e. taking medications, appointment reminders, data input into the device etc. All of these features provided a great product that included proficiencies for the healthcare team and health care ownership for the resident.

Any information that is captured by the resident is considered self-reported data. There is a concern of accuracy due to data entry errors that may occur by the resident, as this information is automatically uploaded into the database hosting the medical record [19]. This data is what the healthcare team would review and utilize in providing care to the resident and though the telehealth device prompted questions to the resident to assist with the accurate collection, there is a need for additional methods to be determined so that the healthcare team could decipher which data is accurate [20]. It is also important for some form of communication to be designed to inform the user when information was successfully transmitted or even when it was reviewed by the health care team. This would assist in providing necessary feedback to the resident and not give them a false sense of security [21].

\section{Assumptions}

- Information capture by a device as opposed to a manual entry will have different levels of accuracy. 
- All residents will be over the age of 65 .

- Every individual will have differing levels of experience based on their life experiences, age and gender.

\section{Significance to Residents' Quality of Care}

Quality care for residents can be improved with accurate medical records. When using alternative methods to collect data it is important to establish policies to assist with verifying that the data is correct. Some peripheral devices that can be attached to telemonitoring devices enable error messages to be displayed if it was not collected correctly. Some telemonitoring devices also contain specific ranges that can be determined by the care team. These ranges will assist the health care team to determine accurate data as they can graphically see and compare other measurements that were collected. Other benefits of successfully implemented telemonitoring programs can be demonstrated through cost savings to facilities, residents and reduced hospitalizations $[22,23]$.

\section{Research Questions}

The guiding question here is: How accurate is self reported data in an Electronic Medical Record and what is the effect on personal experience with technology on the input of data? The study will focus on answering the following questions:

How valid is data input from resident's with computer technology experience?

Does age play a factor in accurate usage of the device?

Does gender play a factor in accurate usage of the device?

\section{Conclusion}

Based on current clinical processes, implementing a telehealth device may be a great strategy to assist in improving the quality of life for residents. Understanding how factors such as age, gender and experience impact learning, understanding and perception of technology will provide a means of structuring training, developing interfaces and specifying feedback to the users of the technology that can assist with mainstream adoption of these types of technologies.

\section{References}

1. Centers for Medicare and Medicaid: Overview of Telemedicine and Telehealth

2. Soar, J.: The potential of information and communication technologies to support ageing and independent living. Annals of Telecommunications 65(9-10), 479-483 (2010)

3. Darkins, A., Ryan, P., Kobb, R., Foster, L., Edmonson, E., Wakefield, B., Lancaster, A.F.: Care Coordination/Home Telehealth: The Systematic Implementation of Health Informatics, Home Telehealth, and Disease Management to Support the Care of Veteran Patients with Chronic Conditions. Telemedicine and e-Health 14(10), 1118-1126 (2008) 
4. Hu, P., Chau, P., Liu Sheng, O., Kar Yan, T.: Examining the Technology Acceptance Model Using Physician Acceptance of Telemedicine Technology. Journal of Management Information Systems 16(2), 91-112 (1999)

5. Nickelson, D.: Telehealth and the Evolving Health Care System: Strategic Opportunities for Professional Psychology. American Psychological Association 29(6), 527-535 (1998)

6. Friedman, R.H., Kazis, L.E., Jette, A., Smith, M.B., Stollerman, J., Torgerson, J., et al.: A telecommunications system for monitoring and counseling patients with hypertension. Impact on Medication Adherence and Blood Pressure Control. American Journal Hypertension 9(4), 285-292 (1996)

7. Piette, J.D., Weinberger, M., McPhee, S.J.: The effect of automated calls with telephone nurse follow-up on patient-centered outcomes of diabetes care. Medical Care 38(2), 218 $230(2000)$

8. Piette, J.D., Weinberger, M., McPhee, S.J., Mah, C.A., Kraemer, F.B., Crapo, L.M.: Do automated calls with nurse follow-up improve self-care and glycemic control among vul-nerable patients with diabetes? The American Journal of Medicine 108(1), 20-27 (2000)

9. Davis, F.D.: Perceived Usefulness, Perceived ease of Use, and User Acceptance of Information Technology. MIS Quarterly 13(3), 319-340 (1989)

10. Yi, Y., Wu, Z., Tung, L.: How Individual Differences Influence Technology usage Behavior? Toward an Integrated Framework. Journal of Computer Information Systems 46(2), 52-63 (2005)

11. Jay, G.M., Willis, S.L.: Influence of direct computer experience on older adults' attitudes toward computers. Journal of Gerontology 47(4), 250-257 (1992)

12. Webster, J., Martocchio, J.J.: Microcomputer Playfulness: Development of a Measure with Workplace Implications. MIS Quarterly 16(2), 201-266 (1992)

13. Weber, R.: Why don't men ever stop to ask for directions? Gender, Social influence, and their tole in technology acceptance and usage behavior. MIS Quarterly 24(1), 115-139 (2000)

14. Ahuja, M.K., Thatcher, J.B.: Moving Beyond Intentions and Toward the Theory of Trying: Effects of Work Environment and Gender on Post-Adoption Information Technology Use. MIS Quarterly 29(3) (2005)

15. Gefen, D., Straub, D.W.: Gender Differences in the Perception and Use of E-Mail: An Extension to the Technology Acceptance Model. MIS Quarterly 21(4), 389-400 (1997)

16. Czaja, S.J., Sharit, J.: Age differences in attitudes toward computers. The Journals of Geron-tology: Psychological Sciences and Social Sciences 53(5), 329-340 (1998)

17. Hackbarth, G., Grover, V., Yi, M.Y.: Computer Playfulness and Anxiety: Positive and Negative Mediators of the System Experience Effect on Perceived ease of Use. Information \& Management 40(3), 221-232 (2003)

18. Roine, R., Ohinmaa, A., Hailey, D.: Assessing telemedicine: a systematic review of the literature. Canadian Medical Association Journal 165(6), 765-771 (2001)

19. Tisnado, D.M., Adams, J.L., Liu, H., Damberg, C.L., Chen, W.P., Hu, F.A., Carlisle, D.M., Mangione, C.M., Kahn, K.L.: What is the concordance between the medical record and pa-tient self-report as data sources for ambulatory care? Medical Care 44(2), 132-140 (2006)

20. Bhandari, A., Wagner, T.: Self-Reported Utilization of Health Care Services: Improving Measurement and Accuracy. Medical Care Research Review 63(2), 217-235 (2006) 
21. Gurley, K., Norcio, A.F.: A Systematic Review of Technologies Designed to Improve and Assist Cognitive Decline for both the Current and Future Aging Populations. In: Proceedings of the HCI International 2009, The 13th International Conference on HumanComputer Interaction, pp. 156-163 (2009)

22. Noel, H.C., Vogel, D.C., Erdos, J.J., Cornwall, D., Levin, F.: Home Telehealth Reduces Healthcare Costs Telemedicine. Journal and e-Health 10(2), 170-183 (2004)

23. Finkelstein, S.M., Speedie, S.M., Potthoff, S.: Home Telehealth Improves Clinical Outcomes at Lower Cost for Home Healthcare Telemedicine and e-Health, vol. 12(2), pp. 128136 (2006) 